\title{
WEIGHTED SUMS OF SUBEXPONENTIAL RANDOM VARIABLES AND THEIR MAXIMA
}

\author{
YIQING CHEN,* Guangdong University of Technology \\ KAI W. NG, ${ }^{* *}$ The University of Hong Kong \\ QIHE TANG, ${ }^{* * *}$ Concordia University
}

\begin{abstract}
Let $\left\{X_{k}, k=1,2, \ldots\right\}$ be a sequence of independent random variables with common subexponential distribution $F$, and let $\left\{w_{k}, k=1,2, \ldots\right\}$ be a sequence of positive numbers. Under some mild summability conditions, we establish simple asymptotic estimates for the extreme tail probabilities of both the weighted sum $\sum_{k=1}^{n} w_{k} X_{k}$ and the maximum of weighted sums $\max _{1 \leq m \leq n} \sum_{k=1}^{m} w_{k} X_{k}$, subject to the requirement that they should hold uniformly for $n=1,2, \ldots$. Potentially, a direct application of the result is to risk analysis, where the ruin probability is to be evaluated for a company having gross loss $X_{k}$ during the $k$ th year, with a discount or inflation factor $w_{k}$.
\end{abstract}

Keywords: Asymptotics; Matuszewska index; weighted sum; maximum; subexponentiality; tail probability; uniformity; ruin probability; discounted loss

2000 Mathematics Subject Classification: Primary 60E20

Secondary 60G50; 60G70

\section{Introduction}

Let $\left\{X_{k}, k=1,2, \ldots\right\}$ be a sequence of independent and identically distributed (i.i.d.) random variables with common distribution function $F=1-\bar{F}$, and let $\left\{w_{k}, k=1,2, \ldots\right\}$ be a sequence of positive numbers such that the series

$$
S_{\infty}(w)=\sum_{k=1}^{\infty} w_{k} X_{k}
$$

is well defined. We are interested in the tail behavior of the series $S_{\infty}(w)$ and the maximum

$$
M_{(\infty)}(w)=\sup _{m \geq 1} \sum_{k=1}^{m} w_{k} X_{k} .
$$

The distribution of $S_{\infty}(w)$ is of interest because the marginal distribution of any stationary linear process

$$
\sum_{k=-\infty}^{\infty} w_{k} X_{m-k}, \quad m=0, \pm 1, \pm 2, \ldots
$$

\footnotetext{
Received 1 June 2004.

* Postal address: School of Economics and Management, Guangdong University of Technology, East Dongfeng Road 729, Guangzhou 510090, Guangdong, P. R. China. Email address: yiqch@263.net

** Postal address: Department of Statistics and Actuarial Science, The University of Hong Kong, Pokfulam Road, Hong Kong, Email address: kaing@hku.hk

*** Postal address: Department of Mathematics and Statistics, Concordia University, 7141 Sherbrooke Street West, Montreal, Quebec, H4B 1R6, Canada. Email address: qtang@mathstat.concordia.ca
} 
for two-sided sequences $\left\{w_{k}, k=0, \pm 1, \pm 2, \ldots\right\}$ and $\left\{X_{k}, k=0, \pm 1, \pm 2, \ldots\right\}$ can be represented as the distribution of some $S_{\infty}(w)$ of the form (1.1). We can also interpret (1.1) as the discounted value of all future losses of a company if we regard the random variable $X_{k}$ as being the gross loss during the $k$ th year and the coefficient $w_{k}$ as being the discount or inflation factor from year $k$ to the present. In this way, the ultimate maximum $M_{(\infty)}(w)$ can be interpreted as the maximal discounted value of future losses, and the tail probability $\mathrm{P}\left(M_{(\infty)}(w)>x\right)$ can be interpreted as the ultimate ruin probability with initial surplus $x \geq 0$.

Zerner (2002) investigated the integrability of series (1.1), showing how the integrability of $f\left(\left|S_{\infty}(w)\right|\right)$, for some positive increasing function $f$, corresponds to the integrability of $g\left(\left|X_{1}\right|\right)$, for another function $g$.

We investigate the subtle tail behavior of the quantities (1.1) and (1.2). More precisely, assuming that the distribution $F$ is subexponential (see below for the definition), with a certain restriction, we seek a condition on the sequence $\left\{w_{k}, k=1,2, \ldots\right\}$ such that the asymptotic result

$$
\mathrm{P}\left(M_{(\infty)}(w)>x\right) \sim \mathrm{P}\left(S_{\infty}(w)>x\right) \sim \sum_{k=1}^{\infty} \bar{F}\left(\frac{x}{w_{k}}\right)
$$

holds as $x \rightarrow \infty$. Henceforth, all limit relationships are for $x \rightarrow \infty$ unless stated otherwise; for two positive functions $A(\cdot)$ and $B(\cdot)$, we write $A(x) \lesssim B(x)$ if $\lim \sup A(x) / B(x) \leq 1$, write $A(x) \gtrsim B(x)$ if liminf $A(x) / B(x) \geq 1$, and write $A(x) \sim B(x)$ if both statements hold. The desired condition is

$$
\sum_{k=1}^{\infty} w_{k}^{\delta}<\infty
$$

for a relevant constant $0<\delta<1$; see condition (3.1) below. Specifically, this condition is fulfilled when $w_{k}=(1+r)^{-k}$ for $k=1,2, \ldots$ and some $r>0$. This is the case recently considered by Tang (2004) in the actuarial science literature.

For closely related work, we refer the reader to Cline (1983), Resnick (1987), and Davis and Resnick (1988), among others. See also Embrechts et al. (1997, Chapter A3.3) for a short review.

The rest of the paper is organized as follows. In Section 2, we recall some preliminaries about subexponential distributions; in Section 3, we present the main result and its corollaries; and, in Section 4, we prove the main result after preparing some lemmas.

\section{Heavy-tailed distributions}

As in much recent research in the fields of applied probability and risk theory, we here restrict our interest to the case of heavy-tailed random variables. A random variable $X$, or its distribution $F$ satisfying $\bar{F}(x)>0$ for any $x \in(-\infty, \infty)$, is said to be heavy tailed to the right if $\operatorname{Eexp}\{\gamma X\}=\infty$ for any $\gamma>0$.

One of the most important classes of heavy-tailed distributions is the subexponential class \&. By definition, a distribution $F$ on $[0, \infty)$ is subexponential, denoted by $F \in \&$, if

$$
\lim _{x \rightarrow \infty} \frac{\overline{F^{(n)}}(x)}{\bar{F}(x)}=n
$$

for some $n \geq 2$ (or, equivalently, for any $n \geq 2$ ), where $F^{(n)}$ denotes the $n$-fold convolution of $F$. More generally, a distribution $F$ on $(-\infty, \infty)$ is still said to be subexponential if the 
distribution $F^{+}(x)=F(x) \mathbf{1}_{\{0 \leq x<\infty\}}$ is subexponential. It is easy to check that (2.1) still holds for the latter general case. Clearly, for a sequence of i.i.d. random variables $\left\{X_{k}, k=1,2, \ldots\right\}$ with common distribution $F \in \mathcal{S}$, from (2.1) it holds, for each $n \geq 2$, that

$$
\mathrm{P}\left(\sum_{k=1}^{n} X_{k}>x\right) \sim \mathrm{P}\left(\max _{1 \leq k \leq n} X_{k}>x\right) .
$$

Relation (2.2) explains why the class $\&$ can be used to model the sizes of large loss variables.

Closely related are the classes $\mathcal{L}$, of long-tailed distributions, and $\mathcal{D}$, of distributions with dominatedly varying tails. By definition, a distribution $F$ on $(-\infty, \infty)$ belongs to the class $\mathcal{L}$ if the relation

$$
\lim _{x \rightarrow \infty} \frac{\bar{F}(x+y)}{\bar{F}(x)}=1
$$

holds for any $y>0$ (or, equivalently, for some $y>0$ ); $F$ belongs to the class $\mathscr{D}$ if the relation

$$
\limsup _{x \rightarrow \infty} \frac{\bar{F}(v x)}{\bar{F}(x)}<\infty
$$

holds for any $v, 0<v<1$ (or, equivalently, for some $v, 0<v<1$ ). It is well known that

$$
\mathscr{D} \cap \mathcal{L} \subset \& \subset \mathcal{L}
$$

see Embrechts et al. (1997, Chapters 1.3, 1.4, and A3) and references therein. A well-known subclass of the intersection $\mathscr{D} \cap \mathcal{L}$ is $\mathcal{R}$, the class of distributions with regularly varying tails. By definition, a distribution $F$ on $(-\infty, \infty)$ belongs to the class $\mathcal{R}$ if there is some $\alpha \geq 0$ such that the relation

$$
\lim _{x \rightarrow \infty} \frac{\bar{F}(x y)}{\bar{F}(x)}=y^{-\alpha}
$$

holds for any $y>0$. We write $F \in \mathcal{R}_{-\alpha}$ in this case. Note that the class $\mathcal{R}$ is the union of all $\mathcal{R}_{-\alpha}$ over the range $0 \leq \alpha<\infty$. A slightly larger subclass of the intersection $\mathscr{D} \cap \mathcal{L}$ is the so-called extended regularly varying (ERV) class. By definition, a distribution $F$ on $(-\infty, \infty)$ belongs to the class $\operatorname{ERV}(-\alpha,-\beta)$, for some $\alpha$ and $\beta$ with $0 \leq \alpha \leq \beta<\infty$, if the relation

$$
y^{-\beta} \leq \liminf _{x \rightarrow \infty} \frac{\bar{F}(x y)}{\bar{F}(x)} \leq \limsup _{x \rightarrow \infty} \frac{\bar{F}(x y)}{\bar{F}(x)} \leq y^{-\alpha}
$$

holds for any $y>1$. Thus, $\mathcal{R}_{-\alpha}=\operatorname{ERV}(-\alpha,-\alpha)$. Note that the class ERV is the union of all $\operatorname{ERV}(-\alpha,-\beta)$ over the range $0 \leq \alpha \leq \beta<\infty$.

In this paper, we will also consider the distribution class, defined below, that complements the class $\mathcal{R}$ in the extreme case that the index $\alpha$ is equal to $\infty$.

Definition 2.1. Let $F$ be a distribution on $(-\infty, \infty) . F$ is said to be rapidly varying tailed, denoted by $F \in \mathcal{R}_{-\infty}$, if

$$
\lim _{x \rightarrow \infty} \frac{\bar{F}(v x)}{\bar{F}(x)}=0 \quad \text { for each } v>1
$$


We remark that a more general notion, namely rapid variation, has been extensively investigated in the literature; for details we refer the reader to the monographs by de Haan (1970, Chapter 1.2) and Bingham et al. (1987, Chapter 2.4), among others.

Let us recall two significant indices, which are crucial for our purposes. Let $F$ be a distribution on $(-\infty, \infty)$. As was done recently by Tang and Tsitsiashvili (2003), for any $y>0$ we set

$$
\bar{F}_{*}(y)=\liminf _{x \rightarrow \infty} \frac{\bar{F}(x y)}{\bar{F}(x)}, \quad \bar{F}^{*}(y)=\limsup _{x \rightarrow \infty} \frac{\bar{F}(x y)}{\bar{F}(x)},
$$

and then define

$$
\mathbb{J}_{F}^{+}=\inf \left\{-\frac{\log \bar{F}_{*}(y)}{\log y}, y>1\right\}, \quad \mathbb{J}_{F}^{-}=\sup \left\{-\frac{\log \bar{F}^{*}(y)}{\log y}, y>1\right\} .
$$

Following Tang and Tsitsiashvili (2003), we call the quantities $\mathbb{J}_{F}^{+}$and $\mathbb{J}_{F}^{-}$the upper and lower Matuszewska indices of the distribution $F$, respectively. Clearly, if $F \in \mathcal{R}_{-\alpha}$ for some $\alpha$, $0 \leq \alpha \leq \infty$, then $\mathbb{J}_{F}^{ \pm}=\alpha$, and if $F \in \operatorname{ERV}(-\alpha,-\beta)$ for some $\alpha$ and $\beta, 0 \leq \alpha \leq \beta<\infty$, then $\alpha \leq \mathbb{J}_{F}^{-} \leq \mathbb{J}_{F}^{+} \leq \beta$. For more details of the Matuszewska indices, see Bingham et al. (1987, Chapter 2.1) and Cline and Samorodnitsky (1994).

Let $F$ be a distribution with a lower Matuszewska index such that $0<\mathbb{J}_{F}^{-} \leq \infty$. With some simple adjustments to the second inequality of Proposition 2.2.1 of Bingham et al. (1987), we see that, for any $p, 0<p<\mathbb{J}_{F}^{-}$, there are positive constants $C_{1}$ and $x_{0}$ such that the inequality

$$
\frac{\bar{F}(x y)}{\bar{F}(x)} \leq C_{1} y^{-p}
$$

holds uniformly for $x y \geq x \geq x_{0}$. Fixing the variable $x=x_{0}$ in (2.3), we find that the inequality

$$
\frac{\bar{F}\left(x_{0} y\right)}{\bar{F}\left(x_{0}\right)} \leq C_{1} y^{-p}
$$

holds uniformly for $y \geq 1$. Then, by substituting $x=x_{0} y$ into the above we find that, for some constant $C_{2}>0$, the inequality

$$
\bar{F}(x) \leq C_{2} x^{-p}
$$

holds uniformly for $x \geq x_{0}$.

Recently, Konstantinides et al. (2002) introduced the following subclass of subexponential distributions.

Definition 2.2. Let $F$ be a distribution on $(-\infty, \infty)$. We say that $F$ belongs to the class $\mathcal{A}$ if $F$ is subexponential and has a lower Matuszewska index such that $0<\mathbb{J}_{F}^{-} \leq \infty$.

Clearly, $0<\mathbb{J}_{F}^{-} \leq \infty$ if and only if

$$
\limsup _{x \rightarrow \infty} \frac{\bar{F}(x y)}{\bar{F}(x)}<1 \quad \text { for some } y>1 .
$$

The reason for introducing this class is mainly to exclude some very heavy-tailed distributions (for instance those that are slowly varying tailed) from the class $\&$. It is easy to see that

$$
\mathcal{R}_{-\alpha} \subset \mathcal{A} \quad \text { for any } \alpha, 0<\alpha<\infty, \quad \text { and } \quad s \cap \mathcal{R}_{-\infty} \subset \mathcal{A} .
$$




\section{The main result and its corollaries}

Henceforth, for two positive bivariate functions $A(x ; n)$ and $B(x ; n)$, we say that the asymptotic relation $A(x ; n) \sim B(x ; n)$ holds uniformly for $n=1,2, \ldots$ if

$$
\lim _{x \rightarrow \infty} \sup _{n \geq 1}\left|\frac{A(x ; n)}{B(x ; n)}-1\right|=0
$$

that is, for any $\varepsilon>0$ there is some $x(\varepsilon)>0$ such that the two-sided inequality

$$
(1-\varepsilon) B(x ; n) \leq A(x ; n) \leq(1+\varepsilon) B(x ; n)
$$

holds for all $x \geq x(\varepsilon)$ and all $n=1,2, \ldots$ Clearly, the asymptotic relation $A(x ; n) \sim B(x ; n)$ holds uniformly for $n=1,2, \ldots$ if and only if

$$
\limsup _{x \rightarrow \infty} \sup _{n \geq 1} \frac{A(x ; n)}{B(x ; n)} \leq 1 \text { and } \liminf _{x \rightarrow \infty} \inf _{n \geq 1} \frac{A(x ; n)}{B(x ; n)} \geq 1 .
$$

The latter two relations mean, respectively, that $A(x ; n) \lesssim B(x ; n)$ and $A(x ; n) \gtrsim B(x ; n)$ hold uniformly for $n=1,2, \ldots$

Recall that $\left\{X_{k}, k=1,2, \ldots\right\}$ is a sequence of i.i.d. random variables with common distribution function $F$, and that $\left\{w_{k}, k=1,2, \ldots\right\}$ is a sequence of positive numbers. For notational convenience, we write, for each $n=1,2, \ldots$,

$$
S_{n}(w)=\sum_{k=1}^{n} w_{k} X_{k}, \quad M_{(n)}(w)=\max _{1 \leq m \leq n} S_{m}(w), \quad M_{n}(w)=\max _{1 \leq k \leq n} w_{k} X_{k}
$$

for a real number $x$, we write $x^{+}=\max \{x, 0\}$ and $x^{-}=-\min \{x, 0\}$; and, for each $n=$ $1,2, \ldots$, we then write

$$
S_{n}^{+}(w)=\sum_{k=1}^{n} w_{k} X_{k}^{+} .
$$

The main contribution of this paper is the following theorem.

Theorem 3.1. Suppose that $F \in \mathcal{A}$ and

$$
\sum_{k=1}^{\infty} w_{k}^{\delta}<\infty \text { for some } \delta, 0<\delta<\frac{\mathbb{J}_{F}^{-}}{1+\mathbb{J}_{F}^{-}}
$$

where $\mathbb{J}_{F}^{-} /\left(1+\mathbb{J}_{F}^{-}\right)=1$ for $\mathbb{J}_{F}^{-}=\infty$. Uniformly for $n=1,2, \ldots$, we then have

$$
\mathrm{P}\left(M_{(n)}(w)>x\right) \sim \mathrm{P}\left(S_{n}^{+}(w)>x\right) \sim \mathrm{P}\left(M_{n}(w)>x\right) \sim \sum_{k=1}^{n} \mathrm{P}\left(w_{k} X_{k}>x\right) .
$$

If, in addition,

$$
S_{\infty}^{-}(w)=\sum_{k=1}^{\infty} w_{k} X_{k}^{-}<\infty
$$

where the inequality is almost sure, then (3.2) holds with $S_{n}^{+}(w)$ replaced by $S_{n}(w)$.

We suggest that the reader compare (3.2) to (2.2). 
We now verify the almost-sure convergence of the series and maxima involved in (3.2). With the convention $w_{0}=0$, we observe that

$$
0 \leq \max _{0 \leq m<\infty} S_{m}(w) \leq S_{\infty}^{+}(w)
$$

Under condition (3.1), applying the well-known monotone convergence theorem and the $C_{r}$-inequality, we have

$$
\mathrm{E}\left(S_{\infty}^{+}(w)\right)^{\delta} \leq \mathrm{E}\left(X_{1}^{+}\right)^{\delta} \sum_{k=1}^{\infty} w_{k}^{\delta}<\infty
$$

where the finiteness of $\mathrm{E}\left(X_{1}^{+}\right)^{\delta}$ is guaranteed by (2.4), since $\delta<\mathbb{J}_{F}^{-}$. This implies that the series $S_{\infty}^{+}(w)$ and the maximum $\max _{0 \leq m<\infty} S_{m}(w)$ are almost surely finite; hence, the tail probabilities $\mathrm{P}\left(M_{(\infty)}(w)>x\right)$ and $\mathrm{P}\left(S_{\infty}^{+}(w)>x\right)$ are not reduced to trivial constants for $x>0$. A similar explanation can be given for the tail probability $\mathrm{P}\left(M_{\infty}(w)>x\right)$. In addition, the convergence of the series $\sum_{k=1}^{\infty} \mathrm{P}\left(w_{k} X_{k}>x\right)$, for each $x>0$, can also be verified since, by (2.3), we have

$$
\mathrm{P}\left(w_{k} X_{k}>x\right) \leq C_{1} w_{k}^{p} \bar{F}(x)
$$

for some $\delta<p<\mathbb{J}_{F}^{-}$, all $x \geq x_{0}$, and all $k=1,2, \ldots$ such that $w_{k} \leq 1$.

A recent large deviations result of Korshunov (2001) has a similar taste to Theorem 3.1. For a subexponential distribution $F$ with $\int_{0}^{\infty} \bar{F}(y) \mathrm{d} y<\infty$, let

$$
\overline{F_{u}}(x)= \begin{cases}\min \left\{1, \int_{x}^{x+u} \bar{F}(y) \mathrm{d} y\right\}, & x \geq 0, \\ 1, & x<0 .\end{cases}
$$

Clearly, for any $u \in[0, \infty], F_{u}$ defines a standard distribution on $[0, \infty)$. According to the terminology of Korshunov (2001), we say that the distribution $F$ is strongly subexponential if the relation

$$
\overline{F_{u}^{(2)}}(x) \sim 2 \overline{F_{u}}(x)
$$

holds uniformly for $u \in[1, \infty]$. Korshunov's result states that if the common distribution $F$ of the increments of a random walk $S_{n}=\sum_{k=1}^{n} X_{k}$ is strongly subexponential and has a finite mean $-\mu=\mathrm{E} X_{1}<0$, then, uniformly for $n=1,2, \ldots$,

$$
\mathrm{P}\left(\max _{1 \leq k \leq n} S_{k}>x\right) \sim \frac{1}{\mu} \int_{x}^{x+n \mu} \bar{F}(y) \mathrm{d} y \sim \sum_{k=1}^{n} \bar{F}(x+k \mu) .
$$

Compared with Korshunov's result, our Theorem 3.1 successfully establishes a corresponding uniform asymptotic result for the case in which the weights are included.

In the case that each $X_{k}$ has a distribution $F_{k}$ satisfying $\overline{F_{k}}(x) \sim c_{k} \bar{F}(x)$ for some distribution $F \in \delta$ and positive constants $c_{k}, k=1,2, \ldots, \mathrm{Ng}$ et al. (2002, Theorem 2.2) established a result similar to (3.2), but without weights and only for a fixed integer $n=1,2, \ldots$.

Clearly, the uniformity of the asymptotics in (3.2) allows for $n=\infty$. Hence, we have the following corollary.

Corollary 3.1. Write $P_{1}(x)=\mathrm{P}\left(M_{(\infty)}(w)>x\right), P_{2}(x)=\mathrm{P}\left(S_{\infty}^{+}(w)>x\right)$, and $P_{3}(x)=$ $\mathrm{P}\left(M_{\infty}(w)>x\right)$ for $x \geq 0$. Under the conditions of Theorem 3.1, we then have

$$
P_{1}(x) \sim P_{2}(x) \sim P_{3}(x) \sim \sum_{k=1}^{\infty} \mathrm{P}\left(w_{k} X_{k}>x\right) .
$$


Specifically, if we choose $w_{k}=(1+r)^{-k}, k=1,2, \ldots$, with some $r>0$, then the relation corresponding to the probability $P_{1}(x)$, which can be interpreted as the ultimate ruin probability of a discrete-time model with constant interest rate $r>0$, coincides with the main result of Tang (2004).

We now put forward some special cases of Corollary 3.1 (the same thing can be done for Theorem 3.1).

Corollary 3.2. Let $P_{i}(x), i=1,2,3$, be as defined in Corollary 3.1.

1. Suppose that $F \in \&$ with $0<\mathbb{J}_{F}^{-} \leq \mathbb{J}_{F}^{+}<\infty$ (or, equivalently, that $F \in \mathscr{D} \cap \mathcal{A}$ ) and that condition (3.1) holds. Then, for $i=1,2,3$,

$$
0<\sum_{k=1}^{\infty} \bar{F}_{*}\left(w_{k}^{-1}\right) \leq \liminf _{x \rightarrow \infty} \frac{P_{i}(x)}{\bar{F}(x)} \leq \limsup _{x \rightarrow \infty} \frac{P_{i}(x)}{\bar{F}(x)} \leq \sum_{k=1}^{\infty} \bar{F}^{*}\left(w_{k}^{-1}\right)<\infty .
$$

2. Suppose that $F \in \operatorname{ERV}(-\alpha,-\beta)$ for some $\alpha$ and $\beta, 0<\alpha \leq \beta<\infty$, and that

$$
\sum_{k=1}^{\infty} w_{k}^{\delta}<\infty \text { for some } \delta, 0<\delta<\frac{\alpha}{1+\alpha} \text {. }
$$

Then, for $i=1,2,3$,

$$
\bar{F}(x) \sum_{k=1}^{\infty} \min \left\{w_{k}^{\alpha}, w_{k}^{\beta}\right\} \lesssim P_{i}(x) \lesssim \bar{F}(x) \sum_{k=1}^{\infty} \max \left\{w_{k}^{\alpha}, w_{k}^{\beta}\right\} .
$$

3. Suppose that $F \in \mathcal{R}_{-\alpha}$ for some $\alpha, 0<\alpha<\infty$, and that condition (3.7) holds. Then, for $i=1,2,3$,

$$
P_{i}(x) \sim \bar{F}(x) \sum_{k=1}^{\infty} w_{k}^{\alpha} .
$$

4. Suppose that $F \in \delta \cap \mathcal{R}_{-\infty}$ and that

$$
\sum_{k=1}^{\infty} w_{k}^{\delta}<\infty \text { for some } \delta, 0<\delta<1 .
$$

Then, for $i=1,2,3$,

$$
P_{i}(x) \sim \bar{F}\left(\frac{x}{w^{*}}\right) \sum_{k=1}^{\infty} \mathbf{1}_{\left\{w_{k}=w^{*}\right\}},
$$

where $w^{*}=\max \left\{w_{k}, k=1,2, \ldots\right\}$.

Proof. Part 3 is clearly a consequence of part 2, which is itself a consequence of part 1 since $\alpha \leq \mathbb{J}_{F}^{-} \leq \mathbb{J}_{F}^{+} \leq \beta$ for $F \in \operatorname{ERV}(-\alpha,-\beta)$. In order to prove part 1, we consider the right-hand side of (3.5). By (2.3) with $p=\delta<\mathbb{J}_{F}^{-}$, (3.4) holds for all $x \geq x_{0}$ and all $k=1,2, \ldots$ such that $w_{k} \leq 1$. Hence, from Fatou's lemma, the application of which is justified by (3.1), we obtain

$$
\limsup _{x \rightarrow \infty} \frac{P_{i}(x)}{\bar{F}(x)} \leq \sum_{k=1}^{\infty} \limsup _{x \rightarrow \infty} \frac{\mathrm{P}\left(w_{k} X_{k}>x\right)}{\bar{F}(x)}=\sum_{k=1}^{\infty} \bar{F}^{*}\left(w_{k}^{-1}\right) .
$$

The corresponding lower bound in (3.6) can be proved similarly. 
We now prove part 4 . The convergence of the series $\sum_{k=1}^{\infty} \mathbf{1}_{\left\{w_{k}=w^{*}\right\}}$ is implied by (3.1). We divide the right-hand side of (3.5) into two parts, as follows:

$$
\sum_{k=1}^{\infty} \mathrm{P}\left(w_{k} X_{k}>x\right)=\left(\sum_{\left\{k: w_{k}=w^{*}\right\}}+\sum_{\left\{k: w_{k}<w^{*}\right\}}\right) \mathrm{P}\left(w_{k} X_{k}>x\right)=: \sum_{1}+\sum_{2} .
$$

Recalling Definition 2.1, by applying Fatou's lemma, as in the proof of part 1, we have

$$
\limsup _{x \rightarrow \infty} \frac{\sum_{2}}{\mathrm{P}\left(w^{*} X_{1}>x\right)} \leq \sum_{\left\{k: w_{k}<w^{*}\right\}} \limsup _{x \rightarrow \infty} \frac{\mathrm{P}\left(w_{k} X_{k}>x\right)}{\mathrm{P}\left(w^{*} X_{1}>x\right)}=0 .
$$

Hence,

$$
\sum_{k=1}^{\infty} \mathrm{P}\left(w_{k} X_{k}>x\right) \sim \sum_{1}=\bar{F}\left(\frac{x}{w^{*}}\right) \sum_{k=1}^{\infty} \mathbf{1}_{\left\{w_{k}=w^{*}\right\}} .
$$

This ends the proof of Corollary 3.2.

Work closely related to Corollaries 3.1 and 3.2 can be found in Cline (1983), Davis and Resnick (1988), and Embrechts et al. (1997, Chapter A3.3). Among them, in the case in which the tail probability $\mathrm{P}\left(\left|X_{k}\right|>x\right)$ is regularly varying at infinity and the weights $\left\{w_{k}, k=\right.$ $1,2, \ldots\}$ are not necessarily positive, Cline (1983) established a result similar to Corollary 3.2.3 but without $P_{1}(x)$. In the case in which the common distribution $F$ is both in the domain of attraction of $\Lambda(x)=\exp \left\{-\mathrm{e}^{-x}\right\}, x \in(-\infty, \infty)$, and in the class $s(\gamma), \gamma \geq 0$, Davis and Resnick (1988) established a result similar to Corollary 3.2.4. Note that the fact that distribution $F$ is in the domain of attraction of $\Lambda(x)$ indicates that $F \in \mathcal{R}_{-\infty}$.

\section{Proof of the main result}

\subsection{Lemmas}

Lemma 4.1. Consider the convolution of two distributions $F_{1}$ and $F_{2}$ on $(-\infty, \infty)$. If $F_{1} \in \delta$ and $\overline{F_{2}}(x) \lesssim c \overline{F_{1}}(x)$, for some $c \geq 0$, then

$$
\overline{F_{1} * F_{2}}(x) \lesssim(1+c) \overline{F_{1}}(x) .
$$

Proof. See Lemma 4.4 of Tang (2004).

Lemma 4.2. Let $\left\{X_{k}, k=1,2, \ldots, n\right\}$ be $n$ independent, real-valued random variables. If each $X_{k}$ has a distribution $F_{k} \in \mathcal{L}, k=1,2, \ldots, n$, then

$$
\mathrm{P}\left(\max _{1 \leq m \leq n} \sum_{k=1}^{m} X_{k}>x\right) \sim \mathrm{P}\left(\sum_{k=1}^{n} X_{k}>x\right) .
$$

Proof. See Theorem 2.1 of $\mathrm{Ng}$ et al. (2002).

Lemma 4.3. Let $\left\{X_{k}, k=1,2, \ldots, n\right\}$ be $n$ i.i.d., real-valued random variables with common distribution $F \in \mathcal{S}$, and let $\left\{w_{k}, k=1,2, \ldots, n\right\}$ be $n$ positive numbers. Then the weighted sum $S_{n}(w)$ is subexponentially distributed and satisfies

$$
\mathrm{P}\left(S_{n}(w)>x\right) \sim \sum_{k=1}^{n} \bar{F}\left(\frac{x}{w_{k}}\right) .
$$


Proof. The proof is based on a result of Tang and Tsitsiashvili (2003); see also Embrechts and Goldie (1980) and Cline (1986, Corollary 1). This result states that if $F_{1} \in \&, F_{2} \in \mathcal{L}$, and $\overline{F_{2}}(x)=O\left(\overline{F_{1}}(x)\right)$, then $F_{1} * F_{2} \in \&$ and

$$
\overline{F_{1} * F_{2}}(x) \sim \overline{F_{1}}(x)+\overline{F_{2}}(x) .
$$

Using this result, we can prove (4.1) for $n=2$ and use induction to prove the general case. This ends the proof of Lemma 4.3.

Lemma 4.4. Under the conditions of Theorem 3.1, we have

$$
\lim _{n \rightarrow \infty} \limsup _{x \rightarrow \infty} \frac{\mathrm{P}\left(\sum_{k=n}^{\infty} w_{k} X_{k}^{+}>x\right)}{\mathrm{P}\left(w_{1} X_{1}>x\right)}=0
$$

and

$$
\lim _{n \rightarrow \infty} \limsup _{x \rightarrow \infty} \frac{\sum_{k=n}^{\infty} \mathrm{P}\left(w_{k} X_{k}>x\right)}{\mathrm{P}\left(w_{1} X_{1}>x\right)}=0 .
$$

Proof. We follow the proofs of Lemma 4.24 of Resnick (1987) and Proposition 1.1 of Davis and Resnick (1988); see also Embrechts et al. (1997, Chapter A3.3) for a simpler treatment.

First, we choose some $p_{1}$ such that

$$
\delta<p_{1}<1-\frac{\delta}{\mathbb{J}_{F}^{-}} .
$$

Then, by (3.1), we have $\sum_{k=1}^{\infty} w_{k}^{p_{1}}<\infty$. For all large $n$ (say $n \geq n_{0}$ for some integer $n_{0}$ such that

$$
\sum_{k=n_{0}}^{\infty} w_{k}^{p_{1}}<1 \quad \text { and } \quad w_{1} w_{k}^{p_{1}-1}>1
$$

for all $k \geq n_{0}$ ), we have

$$
\begin{aligned}
\mathrm{P}\left(\sum_{k=n}^{\infty} w_{k} X_{k}^{+}>x\right) & \leq \mathrm{P}\left(\sum_{k=n}^{\infty} w_{k} X_{k}^{+}>\sum_{k=n}^{\infty} w_{k}^{p_{1}} x\right) \\
& \leq \mathrm{P}\left(\bigcup_{k=n}^{\infty}\left\{w_{k} X_{k}^{+}>w_{k}^{p_{1}} x\right\}\right) \\
& \leq \sum_{k=n}^{\infty} \mathrm{P}\left(w_{1} X_{k}^{+}>w_{1} w_{k}^{p_{1}-1} x\right) .
\end{aligned}
$$

Next, we choose some $p_{2}$ such that

$$
0<\frac{\delta}{1-p_{1}}<p_{2}<\mathbb{J}_{F}^{-} .
$$

Applying (2.3), with $p=p_{2}$, to the right-hand side of (4.4) yields

$$
\sum_{k=n}^{\infty} \mathrm{P}\left(w_{1} X_{k}^{+}>w_{1} w_{k}^{p_{1}-1} x\right) \leq C_{1} \sum_{k=n}^{\infty}\left(w_{1} w_{k}^{p_{1}-1}\right)^{-p_{2}} \mathrm{P}\left(w_{1} X_{1}>x\right)
$$


for all $x \geq x_{0} / w_{1}$. Since $\left(1-p_{1}\right) p_{2}>\delta$, by (3.1) we have

$$
\sum_{k=1}^{\infty}\left(w_{1} w_{k}^{p_{1}-1}\right)^{-p_{2}}<\infty
$$

Hence, (4.4) and (4.5) give the first result (4.2).

Observe that

$$
\sum_{k=n}^{\infty} \mathrm{P}\left(w_{k} X_{k}>x\right) \leq \sum_{k=n}^{\infty} \mathrm{P}\left(w_{1} X_{k}^{+}>w_{1} w_{k}^{p_{1}-1} x\right)
$$

holds for all $x>0$ and all $n$ such that $0<w_{k}<1$ for $k \geq n$. Hence, (4.3) is a natural consequence of (4.5) and the convergence of the series $\sum_{k=1}^{\infty}\left(w_{1} w_{k}^{p_{1}-1}\right)^{-p_{2}}$. This ends the proof of Lemma 4.4.

\subsection{Proof of Theorem 3.1}

We divide the proof into four parts, as follows.

4.2.1. $\mathrm{P}\left(M_{(n)}(w)>x\right) \sim \sum_{k=1}^{n} \mathrm{P}\left(w_{k} X_{k}>x\right)$ holds uniformly for $n$. By Lemma 4.4, for any $\varepsilon, 0<\varepsilon<1$ there are some $x_{0} \equiv x_{0}(\varepsilon)$ and some $m \equiv m(\varepsilon)=1,2, \ldots$ such that

$$
\mathrm{P}\left(\sum_{k=n}^{\infty} w_{k} X_{k}^{+}>x\right) \leq \varepsilon \mathrm{P}\left(w_{1} X_{1}>x\right)
$$

and

$$
\sum_{k=n}^{\infty} \mathrm{P}\left(w_{k} X_{k}>x\right) \leq \varepsilon \mathrm{P}\left(w_{1} X_{1}>x\right)
$$

hold for all $x \geq x_{0}$ and all $n>m$. For this fixed $m$ and each $1 \leq n \leq m$, successive applications of Lemmas 4.2 and 4.3 gives

$$
\mathrm{P}\left(M_{(n)}(w)>x\right) \sim \mathrm{P}\left(S_{n}(w)>x\right) \sim \sum_{k=1}^{n} \mathrm{P}\left(w_{k} X_{k}>x\right) .
$$

Hence, there is some $A \equiv A(\varepsilon)>0$ such that, for all $1 \leq n \leq m$ and $x \geq A$,

$$
(1-\varepsilon) \sum_{k=1}^{n} \mathrm{P}\left(w_{k} X_{k}>x\right) \leq \mathrm{P}\left(M_{(n)}(w)>x\right) \leq(1+\varepsilon) \sum_{k=1}^{n} \mathrm{P}\left(w_{k} X_{k}>x\right) .
$$

By Lemma 4.3, (4.8) further indicates that the partial sum $S_{m}(w)$ and the maximum $M_{(m)}(w)$ are subexponentially distributed.

Now we consider the case that $n>m$. By (4.9), for $x \geq A$, we have

$$
\begin{aligned}
\mathrm{P}\left(M_{(n)}(w)>x\right) & \geq \mathrm{P}\left(M_{(m)}(w)>x\right) \\
& \geq(1-\varepsilon) \sum_{k=1}^{m} \mathrm{P}\left(w_{k} X_{k}>x\right) \\
& =(1-\varepsilon)\left(\sum_{k=1}^{n}-\sum_{k=m+1}^{n}\right) \mathrm{P}\left(w_{k} X_{k}>x\right) .
\end{aligned}
$$


Also, by (4.7), for all $n>m$ and all $x \geq x_{0}$, we have

$$
\sum_{k=m+1}^{n} \mathrm{P}\left(w_{k} X_{k}>x\right) \leq \sum_{k=m+1}^{\infty} \mathrm{P}\left(w_{k} X_{k}>x\right) \leq \varepsilon \mathrm{P}\left(w_{1} X_{1}>x\right) .
$$

Therefore, for all $n>m$ and all $x \geq x_{0}$,

$$
\begin{aligned}
\mathrm{P}\left(M_{(n)}(w)>x\right) & \geq(1-\varepsilon)\left(\sum_{k=1}^{n} \mathrm{P}\left(w_{k} X_{k}>x\right)-\varepsilon \mathrm{P}\left(w_{1} X_{1}>x\right)\right) \\
& \geq(1-\varepsilon)^{2} \sum_{k=1}^{n} \mathrm{P}\left(w_{k} X_{k}>x\right) .
\end{aligned}
$$

By symmetry, we can also derive upper bounds for $\mathrm{P}\left(M_{(n)}(w)>x\right)$ : for all $n>m$,

$$
\begin{aligned}
\mathrm{P}\left(M_{(n)}(w)>x\right) & \leq \mathrm{P}\left(M_{(m)}(w)+\sum_{k=m+1}^{n} w_{k} X_{k}^{+}>x\right) \\
& \leq \mathrm{P}\left(M_{(m)}(w)+\sum_{k=m+1}^{\infty} w_{k} X_{k}^{+}>x\right) .
\end{aligned}
$$

Since, by (4.6),

$$
\mathrm{P}\left(\sum_{k=m+1}^{\infty} w_{k} X_{k}^{+}>x\right) \leq \varepsilon \mathrm{P}\left(M_{(m)}(w)>x\right),
$$

by applying Lemma 4.1 and (4.9), we find that

$$
\begin{aligned}
\mathrm{P}\left(M_{(n)}(w)>x\right) & \lesssim(1+\varepsilon) \mathrm{P}\left(\max _{1 \leq n \leq m} \sum_{k=1}^{n} w_{k} X_{k}>x\right) \\
& \leq(1+\varepsilon)^{2} \sum_{k=1}^{m} \mathrm{P}\left(w_{k} X_{k}>x\right) \\
& \leq(1+\varepsilon)^{2} \sum_{k=1}^{n} \mathrm{P}\left(w_{k} X_{k}>x\right)
\end{aligned}
$$

uniformly for $n>m$. Combining (4.10) and (4.11) gives, uniformly for $n>m$,

$$
(1-\varepsilon)^{2} \sum_{k=1}^{n} \mathrm{P}\left(w_{k} X_{k}>x\right) \lesssim \mathrm{P}\left(M_{(n)}(w)>x\right) \lesssim(1+\varepsilon)^{2} \sum_{k=1}^{n} \mathrm{P}\left(w_{k} X_{k}>x\right) .
$$

From (4.9), we conclude that the two-sided inequality (4.12) actually holds uniformly for $n=1,2, \ldots$. Hence, the uniformity of

$$
\mathrm{P}\left(M_{(n)}(w)>x\right) \sim \sum_{k=1}^{n} \mathrm{P}\left(w_{k} X_{k}>x\right)
$$

follows from the arbitrariness of $\varepsilon>0$. 
4.2.2. $\mathrm{P}\left(S_{n}^{+}(w)>x\right) \sim \sum_{k=1}^{n} \mathrm{P}\left(w_{k} X_{k}>x\right)$ holds uniformly for $n$. Observe that, in the first step, we have proved the uniformity of relation (4.13) with respect to $n=1,2, \ldots$ Applying this to the sequence $\left\{X_{k}^{+}, k=1,2, \ldots\right\}$ yields the desired result immediately.

4.2.3. $\mathrm{P}\left(M_{n}(w)>x\right) \sim \sum_{k=1}^{n} \mathrm{P}\left(w_{k} X_{k}>x\right)$ holds uniformly for $n$. Trivially, for all $n=$ $1,2, \ldots$ and all $x>0$,

$$
\mathrm{P}\left(M_{n}(w)>x\right) \leq \sum_{k=1}^{n} \mathrm{P}\left(w_{k} X_{k}>x\right) .
$$

It remains to prove the reverse inequality. To this end, we shall apply an elementary inequality stating that, for $n$ general events $E_{1}, E_{2}, \ldots, E_{n}$,

$$
\mathrm{P}\left(\bigcup_{k=1}^{n} E_{k}\right) \geq \sum_{k=1}^{n} \mathrm{P}\left(E_{k}\right)-\sum_{1 \leq k \neq l \leq n} \mathrm{P}\left(E_{k} E_{l}\right) .
$$

From this inequality, we find that, for all $n=1,2, \ldots$ and all $x>0$,

$$
\begin{aligned}
\mathrm{P}\left(M_{n}(w)>x\right) & \geq \sum_{k=1}^{n} \mathrm{P}\left(w_{k} X_{k}>x\right)-\sum_{1 \leq k \neq l \leq n} \mathrm{P}\left(w_{k} X_{k}>x, w_{l} X_{l}>x\right) \\
& \geq \sum_{k=1}^{n} \mathrm{P}\left(w_{k} X_{k}>x\right)-\left(\sum_{k=1}^{n} \mathrm{P}\left(w_{k} X_{k}>x\right)\right)^{2} \\
& \geq \sum_{k=1}^{n} \mathrm{P}\left(w_{k} X_{k}>x\right)\left(1-\sum_{k=1}^{\infty} \mathrm{P}\left(w_{k} X_{k}>x\right)\right) .
\end{aligned}
$$

Relation (4.3) trivially implies that $\sum_{k=1}^{\infty} \mathrm{P}\left(w_{k} X_{k}>x\right)$ tends to 0 as $x \rightarrow \infty$. Hence, we obtain the desired result, namely that, uniformly for $n=1,2, \ldots$,

$$
\mathrm{P}\left(M_{n}(w)>x\right) \gtrsim \sum_{k=1}^{n} \mathrm{P}\left(w_{k} X_{k}>x\right) .
$$

4.2.4. Under (3.3), $\mathrm{P}\left(S_{n}(w)>x\right) \sim \sum_{k=1}^{n} \mathrm{P}\left(w_{k} X_{k}>x\right)$ holds uniformly for $n$. Applying the result proved in Section 4.2.2, we know that the relation

$$
\mathrm{P}\left(S_{n}(w)>x\right) \leq \mathrm{P}\left(S_{n}^{+}(w)>x\right) \sim \sum_{k=1}^{n} \mathrm{P}\left(w_{k} X_{k}>x\right)
$$

holds uniformly for $n=1,2, \ldots$ Thus, it remains to prove the uniformity of the relation

$$
\mathrm{P}\left(S_{n}(w)>x\right) \gtrsim \sum_{k=1}^{n} \mathrm{P}\left(w_{k} X_{k}>x\right) .
$$

We go back to the results of Section 4.2.1. From (4.8), we know that the two sides of relation (4.14) are asymptotically equal to one another for $1 \leq n \leq m$. For $n>m$, we have

$$
\mathrm{P}\left(S_{n}(w)>x\right) \geq \mathrm{P}\left(S_{m}(w)-\sum_{k=m+1}^{n} w_{k} X_{k}^{-}>x\right) \geq \mathrm{P}\left(S_{m}(w)-\sum_{k=m+1}^{\infty} w_{k} X_{k}^{-}>x\right) .
$$


Note that, under condition (3.3), the series $U_{m}^{-}(w):=\sum_{k=m+1}^{\infty} w_{k} X_{k}^{-}$is a well-defined nonnegative random variable, the random variables $S_{m}(w)$ and $U_{m}^{-}(w)$ are independent, and the partial sum $S_{m}(w)$ is long tailed. Applying the dominated convergence theorem, we obtain

$$
\mathrm{P}\left(S_{m}(w)-\sum_{k=m+1}^{\infty} w_{k} X_{k}^{-}>x\right) \sim \mathrm{P}\left(S_{m}(w)>x\right) .
$$

Hence, for any $\varepsilon>0$ and $n>m$, by applying (4.8) we have

$$
\begin{aligned}
\mathrm{P}\left(S_{n}(w)>x\right) & \gtrsim \sum_{k=1}^{m} \mathrm{P}\left(w_{k} X_{k}>x\right) \\
& \geq\left(\sum_{k=1}^{n}-\sum_{k=m+1}^{\infty}\right) \mathrm{P}\left(w_{k} X_{k}>x\right) \\
& \gtrsim(1-\varepsilon) \sum_{k=1}^{n} \mathrm{P}\left(w_{k} X_{k}>x\right),
\end{aligned}
$$

as in Section 4.2.1. By the arbitrariness of $\varepsilon>0$, we conclude that (4.14) holds uniformly for $n=1,2, \ldots$ This ends the proof of Theorem 3.1 .

\section{References}

Bingham, N. H., Goldie, C. M. and Teugels, J. L. (1987). Regular Variation. Cambridge University Press.

Cline, D. B. H. (1983). Infinite series of random variables with regularly varying tails. Tech. Rep. 83-24, Institute of Applied Mathematics and Statistics, University of British Columbia.

Cline, D. B. H. (1986). Convolution tails, product tails and domains of attraction. Prob. Theory Relat. Fields 72, $529-557$.

Cline, D. B. H. ANd SAmorodnitsky, G. (1994). Subexponentiality of the product of independent random variables. Stoch. Process. Appl. 49, 75-98.

DAVIS, R. AND RESNICK, S. (1988). Extremes of moving averages of random variables from the domain of attraction of the double exponential distribution. Stoch. Process. Appl. 30, 41-68.

De HaAn, L. (1970). On Regular Variation and Its Application to the Weak Convergence of Sample Extremes (Math. Centre Tracts 32). Mathematisch Centrum, Amsterdam.

Embrechts, P. AND Goldie, C. M. (1980). On closure and factorization properties of subexponential and related distributions. J. Austral. Math. Soc. Ser. A 29, 243-256.

Embrechts, P., Klüppelberg, C. And Mikosch, T. (1997). Modelling Extremal Events for Insurance and Finance. Springer, Berlin.

Konstantinides, D., TAng, Q. And Tsitsiashvili, G. (2002). Estimates for the ruin probability in the classical risk model with constant interest force in the presence of heavy tails. Insurance Math. Econom. 31, 447-460.

Korshunov, D. A. (2001). Large deviation probabilities for the maxima of sums of independent summands with a negative mean and a subexponential distribution. Teor. Veroyatnost. i Primenen. 46, 387-397 (in Russian). English translation: Theory Prob. Appl. 46 (2002), 355-366.

NG, K. W., TANG, Q. And YAng, H. (2002). Maxima of sums of heavy-tailed random variables. Astin Bull. 32, 43-55.

ReSnick, S. I. (1987). Extreme Values, Regular Variation, and Point Processes. Springer, New York.

TANG, Q. (2004). The ruin probability of a discrete time risk model under constant interest rate with heavy tails. Scand. Actuarial J., 229-240.

TANG, Q. AND Tsitsiashvili, G. (2003). Precise estimates for the ruin probability in finite horizon in a discrete-time model with heavy-tailed insurance and financial risks. Stoch. Process. Appl. 108, 299-325.

ZERner, M. P. W. (2002). Integrability of infinite weighted sums of heavy-tailed i.i.d. random variables. Stoch. Process. Appl. 99, 81-94. 